\title{
A novel instrumented outsole for real-time foot kinematics measurement: validation across different speeds and simulated foot landing in Cerebral Palsy gait
}

This paper was downloaded from TechRxiv (https://www.techrxiv.org).

\section{LICENSE}

CC BY-NC-SA 4.0

SUBMISSION DATE / POSTED DATE

$01-10-2021 / 12-10-2021$

\section{CITATION}

Bajpai, Rishabh; Tiwari, Ashutosh; Jain, Anant; Joshi, Deepak (2021): A novel instrumented outsole for realtime foot kinematics measurement: validation across different speeds and simulated foot landing in Cerebral Palsy gait. TechRxiv. Preprint. https://doi.org/10.36227/techrxiv.16718515.v2

$\mathrm{DOI}$ 


\title{
A novel instrumented outsole for real-time foot kinematics measurement: validation across different speeds and simulated foot landing in Cerebral Palsy gait
}

\author{
Rishabh Bajpai, Ashutosh Tiwari, Anant Jain and Deepak Joshi
}

\begin{abstract}
Neuromuscular disorders in Cerebral Palsy (CP) patients lead to foot deformities and affect foot biomechanics leading to compromised gait. Thus, measurement of the foot kinematic measurement is of particular interest to understand and characterize the walking pattern among $C P$ patients. The objective of the present work is to develop a wearable instrument to measure foot kinematics such as foot-to-ground angle in threedimensional planes and to measure the foot clearance i.e., toe and heel clearances. A template-based outsole was developed that incorporated an optical distance sensor located anatomically on the outsole and the magnetometer to measure the foot kinematics. The developed system was validated against the reference marker-based motion capture system (from Noraxon). The data from eight able-bodied participants were acquired simultaneously from both the systems (developed and the reference system) at three different walking speeds. A CoP based feedback was presented to the participants to shift the sagittal $\mathrm{CoP}$ anteriorly, posteriorly and normal to simulate the walking pattern of $\mathbf{C P}$ patients with three different foot landing strategies. Pearson's correlation coefficient of more than or equal to 0.62 , root mean square error of less than or equal to 7.81 degrees and limit of agreement of more than or equal to $95 \%$ is found. Furthermore, a wireless wristband is developed and validated for real-time vibrotactile feedback. The measurement accuracy reported with outsole while participants simulated $\mathrm{CP}$ gait shows the potential of present work in real-time foot kinematics detection in $\mathrm{CP}$ patients. The instrumentation is wearable, low-cost, easy to use and implement.
\end{abstract}

Index Terms-Foot kinematics, Foot clearance, Gait analysis, Wearable systems, Cerebral Palsy, Home healthcare

\section{INTRODUCTION}

$\mathbf{L}$ OCOMOTION is a highly coordinated process involving synchronous movement of various joint angles in threedimensional planes [1]. Gait analysis is required to quantitatively understand the locomotion patterns of the deviated gait especially among the clinical population such as cerebral palsy, stroke, and Parkinson's patients. Due to the compromised motor activity among these populations, gait performance reduces, which could lead to the problems such as reduced walking efficiency [2], long-term musculoskeletal issues and asymmetrical joint loading leading to bone deformities such

R. Bajpai, A. Tiwari and D. Joshi are with Centre for Biomedical Engineering, Indian Institute of Technology Delhi,110016, India, and All India Institute of Medical Science Delhi, New Delhi,110029, India. E-mail: \{bmz208129@iitd.ac.in, rishabhbajpai24@gmail.com\}, joshid@iitd.ac.in.

A. Jain is with Department of Electrical Engineering, Indian Institute of Technology Delhi,110016, India as osteoarthritis [2]. In addition to quantifying the lower limb joint kinematics of the human gait, foot kinematic measurement is more important in many instances. Foot kinematics i.e., foot angles with the walking surface and foot clearance estimation during locomotion play an important role in the field of sports sciences, industrial humanoid robots and general clinical gait analysis [3]. For example, impact on the foot and ankle injury while running depends on the foot angles at foot landing [4], whereas tripping and slippages lead to potential falls depend on the foot angles and the foot clearances [5], [6]. In the past, foot kinematics measurements using 3D markerbased motion capture system has been extensively explored with a high accuracy [7]. Despite high accuracy of these systems, they face few limitations such as these systems are restricted to laboratory data collection, high cost and requires skilled personnel to operate them [8]. Therefore, it is important to record the foot kinematics using wearable instrumentation that can be used in the open space without confined walking in the limited space, is available at low cost, and are easy to use and implement.

The methods used to track the foot trajectory mostly exploit IMU, ultrasound sensor, Infrared (IR) sensor, laser sensor, and optical sensors. For better performance, some of the sensors mentioned above are used simultaneously. A. Peruzzi et al. estimated stride length in level walking using an IMU mounted on the foot [9]. A similar approach was used by Xiaoping Yun et al. for predicting orientation, position, velocity, acceleration, and gait phase values from IMU data [10]. However, the performance of prediction can be improved by integrating multiple sensors. An appreciable work of Arsh Arami et al. includes the estimation of foot orientation and foot clearance [11] using IMU and IR sensors. Christopher Moufawad el Achkar et al. tested an IMU and force sensor based instrumented shoe for real-time activity monitoring [12]. Fokke B. van Meulen et al. interpreted various gait-related kinematics from an outsole mounded IMU [13]. Similarly, many other studies focused on the exploitation of IMU for the prediction of gait related parameters [14]-[24]. IMUbased methods for tracking foot trajectory and estimating the orientation of the foot are ubiquitous. However, there are four major limitations of these methods. First, they are less accurate and prone to various experimental noises such as motion artefacts and imprecise IMU placement [25], [26]. Second, their implementation is difficult due to the complex 
computations needed for calculating distance [25], [26]. Third, most of these methods assumed the whole foot as a single segment by using a single IMU sensor to calculate foot-related kinematics. Although, a better approximation would consider the foot as two connected segments. A recent work from our group has shown the improved two segment model and its effectiveness in foot kinematics measurement [27]. Fourth, they do not provide an accurate measure of foot clearance as double integration is needed for distance calculation [25], [26]. A better approach would use distance sensors for estimating foot clearance. Yufridin Wahab and Norantanum Abu Bakar monitored the relative position of the foot from the ground by using an ultrasound sensor mounted on the backside of the shoe [28]. The feasibility of the use of IR sensor with IMU for real-time foot clearance and environment estimation in several situations is tested by Takahiro Ishikawa and Toshiyuki Murakami [29]. The use of distance sensors in place of IMU and optical sensors provides a better measurement of foot clearance. Various environmental factors such as roughness of floor, the reflectivity of floor's surface, lighting conditions and position of sensors' placement are crucial factors to be considered during the experiment.

\section{A. Motivation and contribution}

In most of the Cerebral Palsy children, due to various bone and neuromuscular abnormalities such as skeletal deformities, impaired motor control, leg length discrepancy, muscle tone and muscle contractures, their walking patterns get changed. In other words, to compensate for the body's physical limitations, lower limb kinematics gets changed. Toe walking, scissor walking, and crouch gait are some common gait disorders in $\mathrm{CP}$ children. In these walking patterns, the foot clearance of $\mathrm{CP}$ children gets reduced from typically developing (TD) children [30]. In addition, in many cases of gait abnormalities in $\mathrm{CP}$ children, progression angle gets affected [31], [32]. Therefore, we need a dedicated device to accurately record foot kinematics for diagnosis and tracking improvements during rehabilitation. Unfortunately, there is no wearable device to accurately measure all three orientations of foot, toe clearance, and heel clearance. This motivated us to propose a wearable outsole for recording foot kinematics. CP patients exhibit different foot striking patterns depending on the cases. Most commonly observed foot landing pattern in $\mathrm{CP}$ patients during foot landing is toe strike (also known as toe walking) [33]. During toe walking patterns CP patients strike the foot on the toe and remain on the toe during the entire stance phase. However, other forms of foot landing pattern were also observed in the $\mathrm{CP}$ patients, i.e. flatfoot landing and rearfoot landing. Therefore, in this study, we simulated different foot striking strategies to observe the measurement accuracy and its potential to work faithfully during different types of foot landing patterns. To this end, we designed the centre of pressure based visual feedback system to motivate the able-bodied individuals to simulate the foot landing strategies similar to the $\mathrm{CP}$ patients. The fact that displacement/shift in the COP during the foot landing defines the foot striking pattern [34]-[36], we, therefore, provided the COP shift feedback during the foot landing to simulate various foot landing patterns. A heel striking foot landing pattern shifts the COP posteriorly, flat foot striking foot landing shifts the COP near to the middle of the foot whereas the toe striking foot landing shifts the COP anteriorly.

Some of the significant contributions of the present work are as follows:

- A wearable, affordable, adjustable, wireless, instrumented outsole is presented for monitoring foot kinematics.

- Development of a CoP-based visual feedback scheme to simulate three foot landing strategies in $\mathrm{CP}$

- The proposed outsole is tested at three walking speeds and three simulated foot conditions for clinical applications.

- The feasibility of real-time processing and toe clearance feedback is tested.

The rest of the work is as follows: the methodology used for developing and validating the proposed outsole is explained in Section [I] Experimental results and discussion of the results are presented in Section IIII Finally, the conclusion is drawn in Section IV

\section{Methodology}

\section{A. Instrumentation design and development}

The proposed adjustable outsole incorporates one three-axis magnetometer and six Vertical Cavity Surface-Emitting Laser (VCSEL) at different anatomical locations of the foot as shown in Fig. 1

VCSEL (STMicroelectronics) transmits and records a $940 \mathrm{~nm}$ invisible infrared radiation which is safe for the human eye. It works accurately at various coloured surfaces and different illuminance values with a range is upto $2 \mathrm{~m}$ (according to the datasheet) by using Time-of-Flight (ToF). Six VCSEL are connected to Arduino micro using an Interintegrated circuit (serial bus) interface for device control and data transfer. Since VCSEL follows line-of-sight (LOS), it does not give the actual distances of the sensor from the ground. However, by multiplying the measured distance with the cosine of foot to ground angle (FGA), the actual distances can be computed. For calculating FGA at the forefoot in the sagittal plane, two VCSEL (S1 ans S2 in Fig. 1) separated by a distance ' $\mathrm{L} 1$ ' $(\mathrm{L} 1=48 \mathrm{~mm})$ are used. Let us assume distances calculated by these sensors are ' $\mathrm{d} 1$ ' and ' $\mathrm{d} 2$ ' for actual distances 'D1' and 'D2', respectively. So, the FGA at the forefoot is given by the following equation 1

$$
F G A=\frac{\tan ^{-1}(d 1 / d 1)}{L 1}
$$

D1 and D2 are calculated by the following equations 2 and 3 Here, D1 considered as the toe clearance (TC).

$$
\begin{aligned}
& D 1=d 1 * \cos (F G A) \\
& D 2=d 2 * \cos (F G A)
\end{aligned}
$$

Similarly, for calculating FGA, and actual distances of sensors at hindfoot in the sagittal plane and coronal plane, two sensors along progression direction and two sensors along 


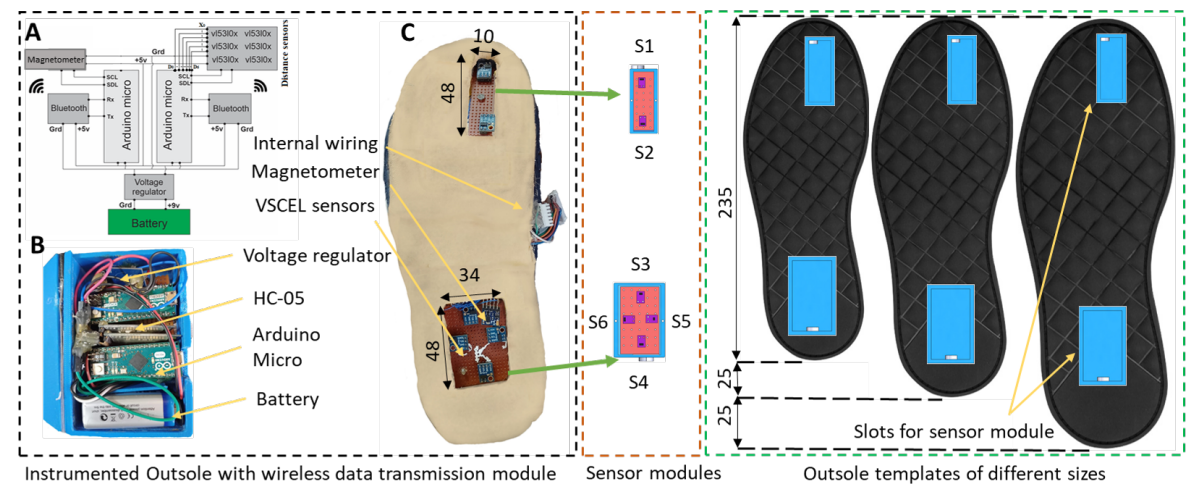

Fig. 1. Black-box: A. circuit diagram of the outsole data transmission system. B. inner view of the outsole data transmission system. C. The proposed Instrumented Outsole with dimensions of sensor modules in mm. Orange-box: CAD model of two sensor modules with the label of sensors. Green-box: Outsole templates of different sizes. Each outsole template has two sensor slots, internal wiring and a connecting socket for the outsole data transmission system. A pair of sensor modules can measure the foot kinematics of users with different shoe sizes by placing them in the slots present in different outsole templates. This design methodology improves the adaptability and affordability of the proposed outsole.

orthogonal to progression direction are used, respectively (see Fig. 1). The distance D6 is considered as the heel clearance (HC). A magnetometer (QST Corporation, Shanghai, China) attached to posterior part of the outsole is used for calculating the yaw angle of the foot.

\section{B. Data Acquisition System}

The data acquisition system (DAS) consists of two modules; data transmission module and data receiving module. Data transmission module acquires the data from the six distance sensors and magnetometer. and simultaneously transmits the data wirelessly through a bluetooth module (HC-05). The internal circuit schematic is shown in the Fig. 1. The circuit consists of the Arduino micro to read the voltage from the sensors, a voltage regulator, a bluetooth module, and a rechargeable battery of $600 \mathrm{mAh}$ (from EBL). A 3D printed box contains the circuit and is mounted at the back of the shoe/sandal. The data transmitted by the transmission module is received and stored on a computer system by the data receiving module. Data receiving module also receives triggers from the video capturing system using myosync (Norxon, USA), for synchronizing the outsole with the video capturing system. The data stream coming from the outsole is stored in the computer system using an open source serial port terminal application (Tera Term, version 4.106) for proper data collection.

\section{Validation with the gold standard}

Eight able-bodied male subjects (mean \pm SD, age: $26 \pm$ 1.6 years, height: $176.6 \pm 7.7 \mathrm{~cm}$ and weight: $74.8 \pm 11.4$ $\mathrm{kg}$ ) having no ambulatory dysfunction and no neurological disorder are recruited for this study. Three experimental protocols including validation at different speeds, COP foot landing strategy at preferred speed and validation of bio-feedback for future applications are followed in each experiment. This study was approved by the All India Institute of Medical Sciences (AIIMS), New Delhi, India ethics committee (Ref. no IEC222/04.05.2018)
1) Experimental protocol 1: Validation at a different walking speed: Each participant was equipped with the instrumented outsole and passive retro-reflective markers of the multi-camera 2D motion capture system (Noraxon, USA) located on the anatomical location on the foot for the validation purpose (as shown in the Fig. 2). Before the data collection, $10 \mathrm{~min}$ of the treadmill acclimatization was provided to the participants. A total of three trials were conducted for each walking speed i.e., slow $(1.8 \mathrm{~km} / \mathrm{h})$, medium $(2.8 \mathrm{~km} / \mathrm{h})$, and fast $(3.8 \mathrm{~km} / \mathrm{h})$. Three trials of 75 seconds are conducted for each walking strategy. Between the trials, $2 \mathrm{~min}$ of the rest was provided. The walking speed was adjusted on the motorized treadmill by the experimenter. The data were recorded simultaneously from the instrumented outsole and the motion capture system for validation purposes.

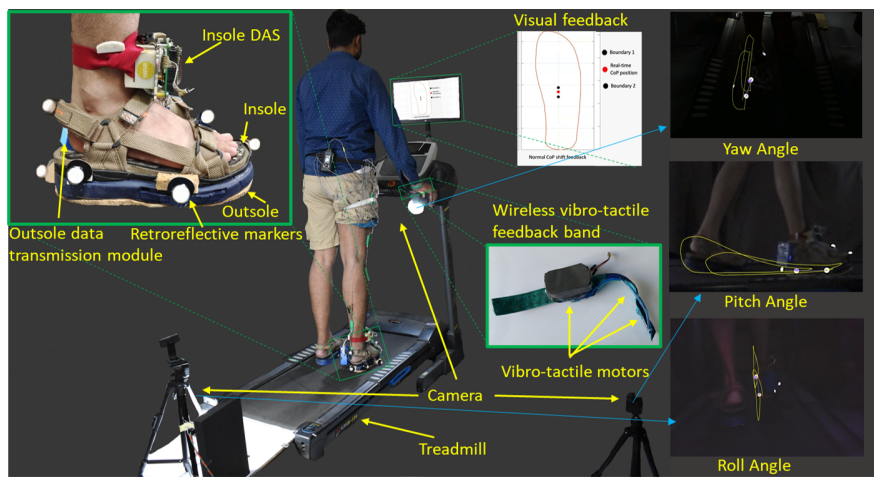

Fig. 2. The figure presents the experimental setup used during the experimental protocol 1, 2 and 3, along with sample trajectories of the recto-reflective markers for one gait cycle.

2) Experimental protocol 2: COP foot landing strategy at preferred speed: A self-developed insole [8] was used for recording vertical ground reaction force at various positions on the foot during the experiment. The insole was equipped with 16 force-sensitive resistors (FlexiForce Standard Model A301, Tekscan, South Boston, USA) with a range of 0-100 lbs $(0-445 \mathrm{~N})$, which is sufficient for plantar pressure measurement applications during static and dynamic loading (as per the datasheet). These ground reaction forces were then used 
for calculating the centre of pressure $(\mathrm{CoP})$. Three different types of foot striking strategies i.e., rearfoot landing (with heel strike), midfoot landing (with flat foot strike), and the forefoot landing (with toe strike) were adopted by the participants while walking at the preferred walking speed on the treadmill. The preferred walking speed was decided based on verbal feedback from the participants. The visual CoP feedback was presented to the participants that motivates the participants to shift the CoP while walking. Moreover, different CoP shift visual feedback was provided corresponding to the different foot landing patterns. Posterior CoP shift feedback corresponds to the rearfoot landing pattern, normal CoP shift feedback corresponds to the midfoot striking pattern, and the anterior CoP shift feedback corresponds to the forefoot landing pattern. Two trials of 90 seconds were conducted for each walking strategy. Where, 2 min of the rest was provided between the trials. CoP data from the insole and the foot kinematics data from the outsole were recorded.

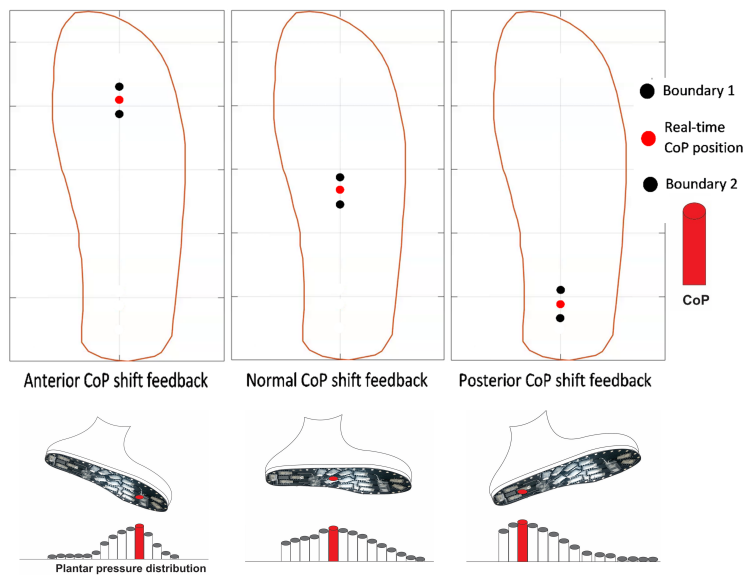

Fig. 3. During the experimental protocol 2, three types of feedbacks are given viz., anterior CoP shift feedback (left), normal CoP shift feedback (middle) and posterior CoP shift feedback (right).

The motivation for validating our outsole at various landing strategies originated from common gait abnormalities in cerebral palsy. Excessive dorsiflexion in crouch gait to spasticity, flat foot due to abnormal contractures and toe walking due to contracture in Achilles tendon may lead to a rearfoot landing, midfoot landing and forefoot landing, respectively (see Fig. 3. Therefore, to validate the applicability of the proposed outsole for cerebral palsy patients, we simulated three abnormal gait patterns.

3) Validation of bio-feedback for future applications: In this protocol, real-time visual feedback for toe trajectory was provided to the subjects during walking at their preferred speed. A self-developed internet of things (IOT) enabled wristband for vibrotactile bio-feedback was integrated with the proposed outsole for providing TC during walking. The wristband consists of ESP8266 nodemcu module, three vibrotactile motors and a rechargeable battery of $600 \mathrm{mAh}$ (from EBL). The instrument outsole wirelessly sends data to DAS, which was then processed and stored to a remote server. The wristband accesses the data from the server and notifies the user whenever the minimum toe clearance (MTC) of the user reduces significantly from his/her normal MTC in three consecutive gait cycles. Minimum Toe Clearance (MTC) is defined as the minimum vertical distance between the toe and the ground surface during the mid-swing phase of the gait cycle [37]. In elderly, lower limb amputee and cerebral palsy populations, low values of MTC are very common [38]. Low MTC value is found to be responsible for falling after tripping in these populations. During the time of MTC, the velocity of the foot is high. Therefore the chances of falling after tripping increases in low MTC conditions [5], [6]. The purpose of this experiment was to test the applicability of the proposed outsole for real-time biofeedback applications. There were two types of trials in this protocol. Fisrt set of trials are normal walking trials and other set of trials are low MTC trials. The subjects were instructed to maintain their MTC to a given range shown by two parallel lines (see Fig. 4). No biofeedback was given in normal walking trial. Whereas, a short 5 second vibrotactile biofeedback was given in low MTC trials.

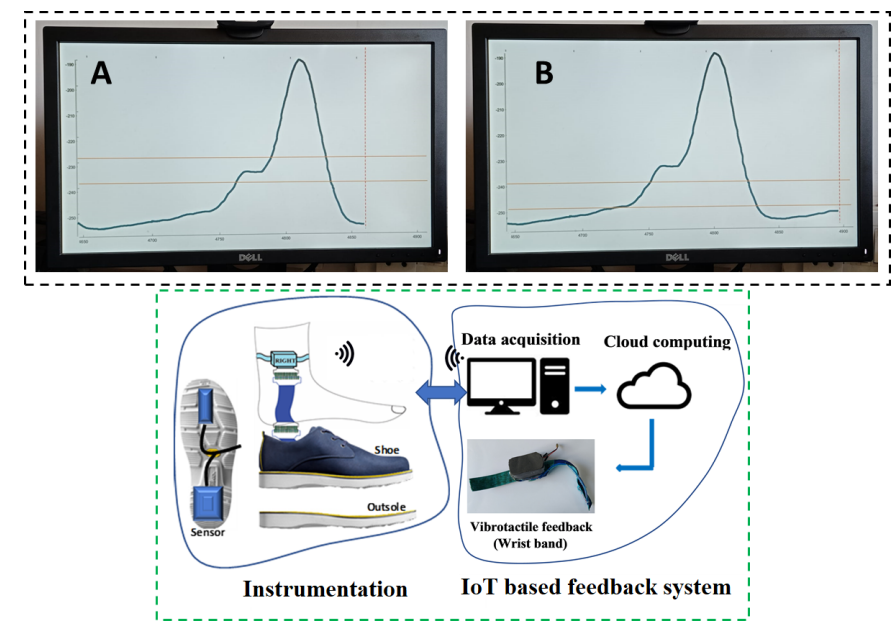

Fig. 4. Black-box: Real-time visual feedback given while following the experiment protocol 3 to motivate the subject to manipulate their MTC. A: A screenshot of visual feedback during a normal MTC walking trial. B: A screenshot of visual feedback during a low MTC walking trial. The red vertical dotted line shows the current gait cycle instance. The two orange horizontal lines show the target range of MTC. The subjects are supposed to shift their MTC according to the target MTC range. Green-box: a pictorial representation of the data flow and its processing during experiment protocol 3 .

\section{Data processing}

For data processing, a system having the following specifications were used; Intel(R) Xeon (R) CPU E3-1225 v5 @ 3.30GHz, 8 GB DDR4 RAM, 64-bit Windows 10 Operating System and Matlab 2020a platform. The raw data stored by DAS of the outsole contains distance values of VCSEL sensors and the three-dimensional intensity values of the magnetic field. As mentioned in the equations 1 and 2, actual values of sensors from the ground were calculated by multiplying the cosine of the angle made by the foot at the point of sensor placement. In addition, the pitch angle $(\alpha)$ and roll angle $(\beta)$ of the foot are calculated by the equations 1 and 2 from sensor 3 (S3) and sensor 4 (S4), and sensor 5 (S5) and sensor 6 (S6) as shown in Fig. 11, respectively. These angles were then used with $\mathrm{x}$ dimensional, $\mathrm{y}$ dimensional and $\mathrm{z}$ dimensional values 
of the magnetometer to calculate the corrected yaw angle $(\gamma$ of the foot as given by equations 4,5 and 6 .

$$
\begin{array}{r}
X=\operatorname{mag}_{x} * \cos (\alpha)+\operatorname{mag}_{y} * \sin (\beta) * \sin (\alpha) \\
+\operatorname{mag}_{z} * \cos (\beta) * \sin (\alpha) \\
Y=\operatorname{mag}_{y} * \cos (\beta)-\operatorname{mag}_{z} * \sin (\beta) \\
\gamma=-\frac{180 * \tan ^{-1}(Y / X)}{\pi}
\end{array}
$$

For each subject, the distance data is calibrated by subtracting individual sensor values with their values at the standing position. In combination with FGA (roll and pitch) and distances of foot from the ground computed from VCSEL, and yaw angle calculated from magnetometer, complete linear and angular foot kinematics can be measured.

A marker-based camera system (one High speed NoraxonTM Ninox 125 camera system with $736 \times 352$ resolution and 125 fps along with two Logitech webcams (B525 and C615 with 720p and $30 \mathrm{fps}$ ) were positioned around the treadmill. All the three cameras were connected to NoraxonTM myoVIDEO software Module on NoraxonTM myoResearchR platform (MR 3.16) for recording the GAIT data. The Ninox 125 camera was placed in the sagittal plane $2 \mathrm{~m}$ away form the centre of the treadmill. The two Logitech webcam were placed in front of and behind the subject at both ends of the treadmill. The cross-section plane of the field of view of the camera placed behind the subject was kept parallel to the coronal plane. Similarly, the cross-section plane of the field of view of camera placed in front of the subject was kept parallel to the transverse plane. The angle measurement was done using the myoResearchR software in the post-processing step. The 2 - point angle measurement option was utilized to capture 2D kinematics by tracking reflective markers throughout the video recording.

For each subject, the distance data is calibrated by subtracting individual sensor values with their values at the standing position. Similarly, the values recorded from the video capturing system were corrected by removing the offset values calculated at the standing position. In addition, these values were scaled by a scaling factor calculated from known distance values. Calculated angles, $\mathrm{HC}$ and $\mathrm{TC}$ from the video capturing system and instrumented outsole were timesynchronized on MATLAB by using markers from triggers. The data was segmented into gait cycles by using COP and vertical ground reaction force (vGRF) values. Using a previous approach, vGRF and CoP data were used to detect gait events, i.e., HS and TO. The gait event instances were computed based on the simple threshold rule using time-series $\operatorname{CoP}(\mathrm{k})$ and time-series $\operatorname{vGRF}(\mathrm{k})$ signals as an input. A vGRF $(\mathrm{k})$ greater than $10 \mathrm{~N}$ and $\mathrm{CoP}(\mathrm{k})$ less than CoPFF (which is the CoP value at flat foot defined as the average of the maximum and minimum CoP value during stance phase) provides the instances of the heel strike. Similarly, toe-off instances were detected when the $\operatorname{vGRF}(\mathrm{k})$ is greater than $10 \mathrm{~N}$ and $\operatorname{CoP}(\mathrm{k})$ is greater than the CoPFF. For further processing, noisy gait cycles were identified and removed manually.

\section{RESULTS AND DISCUSSION}

\section{A. Comparison for angle values}

The calculated roll, pitch and yaw angle by instrumented outsole (IO) and video capturing system (VCS) are compared at three walking speeds i.e. slow, medium and fast, and threefoot landing strategies i.e. rearfoot strike (posterior CoP shift), midfoot strike (normal CoP shift) and forefoot strike (anterior CoP shift). Bland-Altman plot [39], root mean square error (RMSE) and Pearson correlation coefficient (PCC) are calculated at each condition. Fig. 5. A shows sample plots of the foot angles at various walking speeds and foot landing strategies for one subject. Fig. 5B shows sample Bland-Altman plots for foot angles at various walking speeds and foot landing strategies for one subject. Table I presents RMSE, PCC, LoA, for values of the yaw angle measured from the instrumented outsole and video capturing system for all subjects. Similarly, Table III presents PCC, LoA, for values of the pitch angle measured from the IO and VCS for all subjects, and Table III presents RMSE, PCC, LoA, for values of the roll angle measured from the IO and VCS for all subjects. Tables I] II and III depicts that RMSE at each condition is less than or equal to 6.12 and PCC is more than or equal to 0.62. Fig. 5B B shows more than or equal to $95 \%$ difference of values of IO and VCS lies in 95\% confidence level range. These results suggest that both configurations can be used interchangeably for clinical use [39] at various walking speeds.

TABLE I

RMSE, PCC, LOA, FOR VALUES OF THE YAW ANGLE MEASURED FROM THE INSTRUMENTED OUTSOLE AND VIDEO CAPTURING SYSTEM FOR ALL SUBJECTS.

\begin{tabular}{l|l|l|l}
\hline Protocol & RMSE & PCC & LoA \\
\hline Slow & 1.42 & 0.95 & 95.60 \\
Medium & 2.32 & 0.89 & 96.39 \\
Fast & 1.93 & 0.84 & 95.74 \\
Posterior & 2.89 & 0.78 & 95.55 \\
Normal & 1.01 & 0.88 & 95.00 \\
Anterior & 1.68 & 0.82 & 95.52 \\
\hline
\end{tabular}

TABLE II

RMSE, PCC, LOA, FOR VALUES OF THE PITCH ANGLE MEASURED FROM THE INSTRUMENTED OUTSOLE AND VIDEO CAPTURING SYSTEM FOR ALL SUBJECTS .

\begin{tabular}{l|l|l|l}
\hline Protocol & RMSE & PCC & LoA \\
\hline Slow & 4.38 & 0.91 & 98.46 \\
Medium & 3.84 & 0.94 & 96.38 \\
Fast & 5.89 & 0.92 & 96.44 \\
Posterior & 6.12 & 0.80 & 96.73 \\
Normal & 4.79 & 0.97 & 96.38 \\
Anterior & 4.56 & 0.92 & 97.64 \\
\hline
\end{tabular}


TABLE III

RMSE, PCC, LOA, FOR VALUES OF THE ROLL ANGLE MEASURED FROM THE INSTRUMENTED OUTSOLE AND VIDEO CAPTURING SYSTEM FOR ALL SUBJECTS.

\begin{tabular}{l|l|l|l}
\hline Protocol & RMSE & PCC & LoA \\
\hline Slow & 2.32 & 0.72 & 98.78 \\
Medium & 2.54 & 0.69 & 99.21 \\
Fast & 3.85 & 0.62 & 99.63 \\
Posterior & 2.47 & 0.66 & 98.98 \\
Normal & 2.91 & 0.84 & 98.11 \\
Anterior & 3.64 & 0.74 & 96.78 \\
\hline
\end{tabular}

\section{B. Comparison of the $T C$ and $H C$}

The calculated TC and HC by IO and VCS are compared at three walking speeds i.e. slow, medium and fast, and threefoot landing strategies i.e. rearfoot strike (posterior CoP shift), midfoot strike (normal CoP shift) and forefoot strike (anterior CoP shift). Bland-Altman plot [39], RMSE and PCC are calculated at each condition. Fig. 6.A shows sample plots of $\mathrm{TC}$ and $\mathrm{HC}$ at various walking speeds and foot landing strategies for one subject. Fig. 6.B shows sample BlandAltman plots for TC and $\mathrm{HC}$ at various walking speeds and foot landing strategies for one subject. Table IV presents RMSE, PCC, LoA, for values of the TC measured from the IO and VCS for all subjects. Similarly, Table V presents PCC, LoA, for values of the HC measured from the IO and VCS for all subjects. Tables $[\mathrm{IV}$ and $\mathrm{V}$ depicts that RMSE at each condition is less than or equal to 7.81 and PCC is more than or equal to 0.68 . Fig. 6 shows, more than or equal to $95.75 \%$ difference of values of IO and VCS lies in 95\% confidence level range. These results suggest that both configurations can be used interchangeably for clinical use [39] at various walking speeds.

TABLE IV

RMSE, PCC, LOA, FOR VALUES THE TOE CLEARANCE MEASURED FROM THE INSTRUMENTED OUTSOLE AND VIDEO CAPTURING SYSTEM FOR ALL SUBJECTS.

\begin{tabular}{l|l|l|l}
\hline Protocol & RMSE & PCC & LoA \\
\hline Slow & 7.81 & 0.68 & 96.66 \\
Medium & 5.41 & 0.78 & 96.64 \\
Fast & 5.89 & 0.91 & 95.74 \\
Posterior & 6.36 & 0.81 & 96.00 \\
Normal & 5.66 & 0.79 & 97.11 \\
Anterior & 6.83 & 0.68 & 97.77 \\
\hline
\end{tabular}

TABLE V

RMSE, PCC, LOA, FOR VALUES THE HEEL CLEARANCE MEASURED FROM THE INSTRUMENTED OUTSOLE AND VIDEO CAPTURING SYSTEM FOR ALL SUBJECTS.

\begin{tabular}{l|l|l|l}
\hline Protocol & RMSE & PCC & LoA \\
\hline Slow & 6.8 & 0.95 & 98.78 \\
Medium & 6.11 & 0.97 & 97.45 \\
Fast & 7.54 & 0.96 & 98.89 \\
Posterior & 6.12 & 0.97 & 97.15 \\
Normal & 5.32 & 0.98 & 98.56 \\
Anterior & 6.61 & 0.97 & 97.64 \\
\hline
\end{tabular}

At slow walking speed, the mean RMSE values of the angles is 2.71 , mean PCC is 0.86 , and mean LoA is 97.61. For TC and $\mathrm{HC}$ at slow walking speed, the mean of RMSE values of the angles is 7.31, mean PCC is 0.82 , and mean LoA is 97.72 . At medium walking speed, mean RMSE values of the angles is 2.9, mean PCC is 0.84, and mean LoA is 97.33. For TC and $\mathrm{HC}$ at medium walking speed, mean of RMSE values of the angles is 5.76, mean PCC is 0.88 , and mean LoA is 97. At fast walking speed, mean RMSE values of the angles is 3.89, mean PCC is 0.79, and mean LoA is 97.27. For TC and HC at fast walking speed, mean of RMSE values of the angles is 6.72, mean PCC is 0.94 , and mean LoA is 97.32. These results suggest that as walking speed increases, RMSE of angle values increases and the correlation between the IO and VCS decreases. Conversely, as walking speed increases RMSE of distance values decreases (except for medium speed condition) and the correlation between the IO and VCS increases. The possible reason for this could be the increase of range of motion in fast speed when compared with slow speed. As the range of motion of joints increases, rang of the angle of foot increases. Since the camera of VCS records projection of angle profiles in a plane, increasing in range of angle values in other planes can cause errors. In comparison, distance values might not be significantly affected as speed increases.

At posterior CoP shift, mean RMSE values of the angles is 3.83, mean PCC is 0.75 , and mean LoA is 97.09 . For TC and $\mathrm{HC}$ at posterior $\mathrm{CoP}$ shift, mean of RMSE values of the angles is 6.24, mean PCC is 0.89 , and mean LoA is 96.58. At normal CoP shift, mean RMSE values of the angles is 2.83 , mean PCC is 0.90 , and mean LoA is 96.50 . For TC and HC at normal CoP shift, mean of RMSE values of the angles is 5.49, mean PCC is 0.89, and mean LoA is 96.34. At anterior CoP shift, mean RMSE values of the angles is 3.29 , mean PCC is 0.82 , and mean LoA is 96.65 . For TC and HC at anterior CoP shift, mean of RMSE values of the angles is 6.72, mean PCC is 0.83 , and mean LoA is 97.71. In general, the errors of posterior $\mathrm{CoP}$ shift trial and anterior shift trials are more than the normal CoP trials. The possible reason for this could be the same as mentioned above for the increase of error with walking speed.

\section{User-centred real-time biofeedback validation}

To analyse the feasibility of the proposed outsole for a realtime gait correction feedback, a design protocol is followed. A set of verbal questions were asked to the user to qualitatively measure explicit understanding of the users about the biofeedback. The users are satisfied with the responsiveness of the biofeedback and found it to be prospectively useful in certain conditions.

\section{Limitations and the future work}

The distance sensors used within the instrumentation are the optical sensor based on the reflectance and therefore, prone to the measurement inaccuracy due to different colour of the reflected object, surface roughness, and ambient light conditions. In future we would like to exploit the performance of the sensor on various conditions such as varying colours of the walking surface, varying surface roughness, and different ambient light conditions such as different light brightness etc. This understanding would help in the better recommendation 

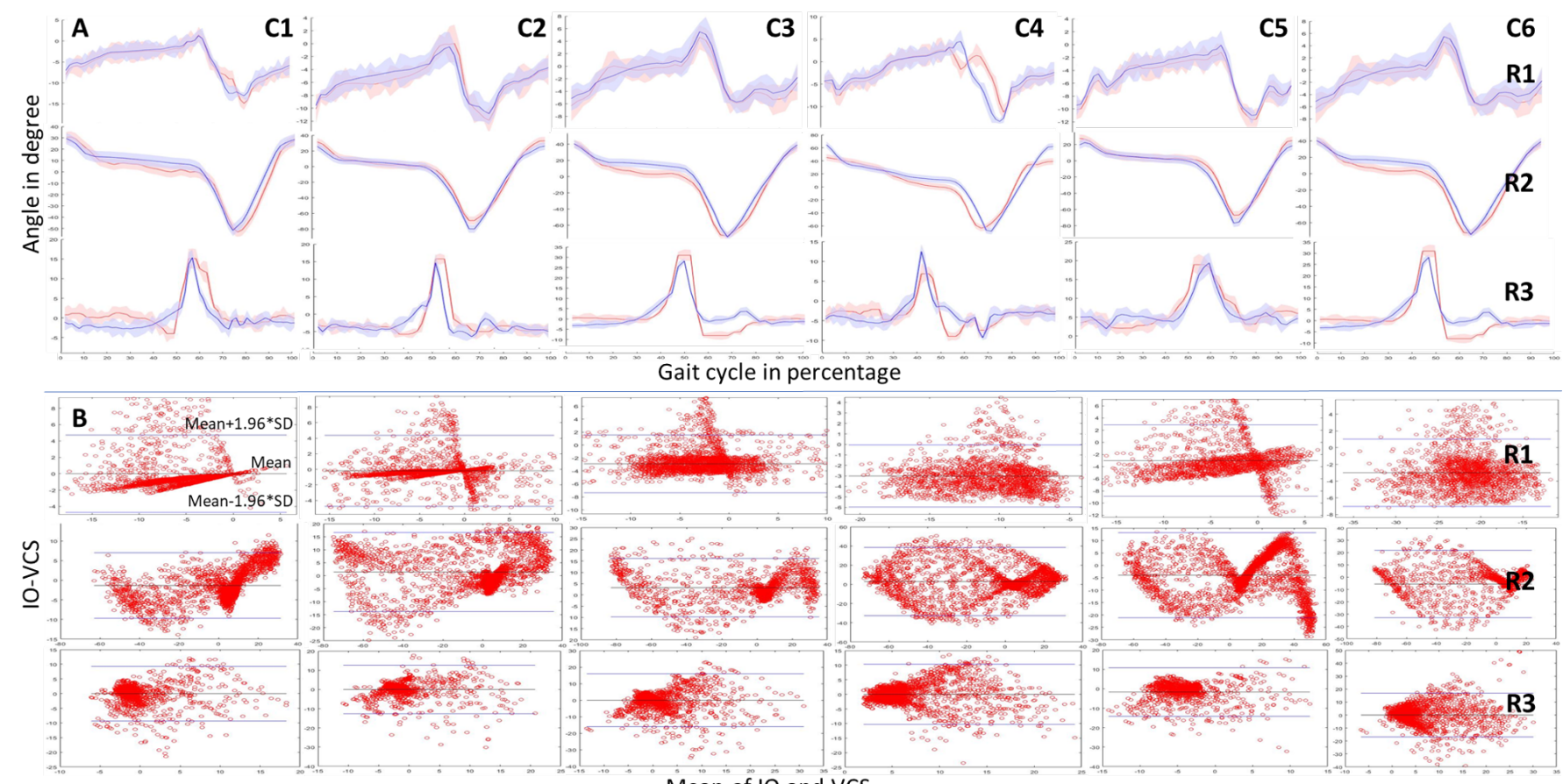

Mean of 10 and VCS

Fig. 5. A: Sample plots of foot angles at various walking speeds and foot landing strategies for one subject. The blue curves correspond to values measured from the video capturing system (VCS), and the red curves correspond to values measured from the instrumented outsole (IO). B: Sample Bland-Altman plots for foot angles at various walking speeds and foot landing strategies for one subject. The Bland-Altman plot is computed for every gait cycle instance throughout the trials. X-value of a point in the plot represents the mean of values measured from IO and VCS. Moreover, the Y-value of a point in the plot represents the difference of values measured from IO and VCS, i.e., IO-VCS. The rows R1, R2 and R3, correspond to yaw angle, pitch angle and roll angle, respectively. The columns $\mathrm{C} 1, \mathrm{C} 2, \mathrm{C} 3, \mathrm{C} 4, \mathrm{C} 5$, and $\mathrm{C} 6$ correspond to slow walking, medium walking, fast walking, posterior CoP shift walking, normal CoP shit walking and anterior CoP shift walking, respectively.
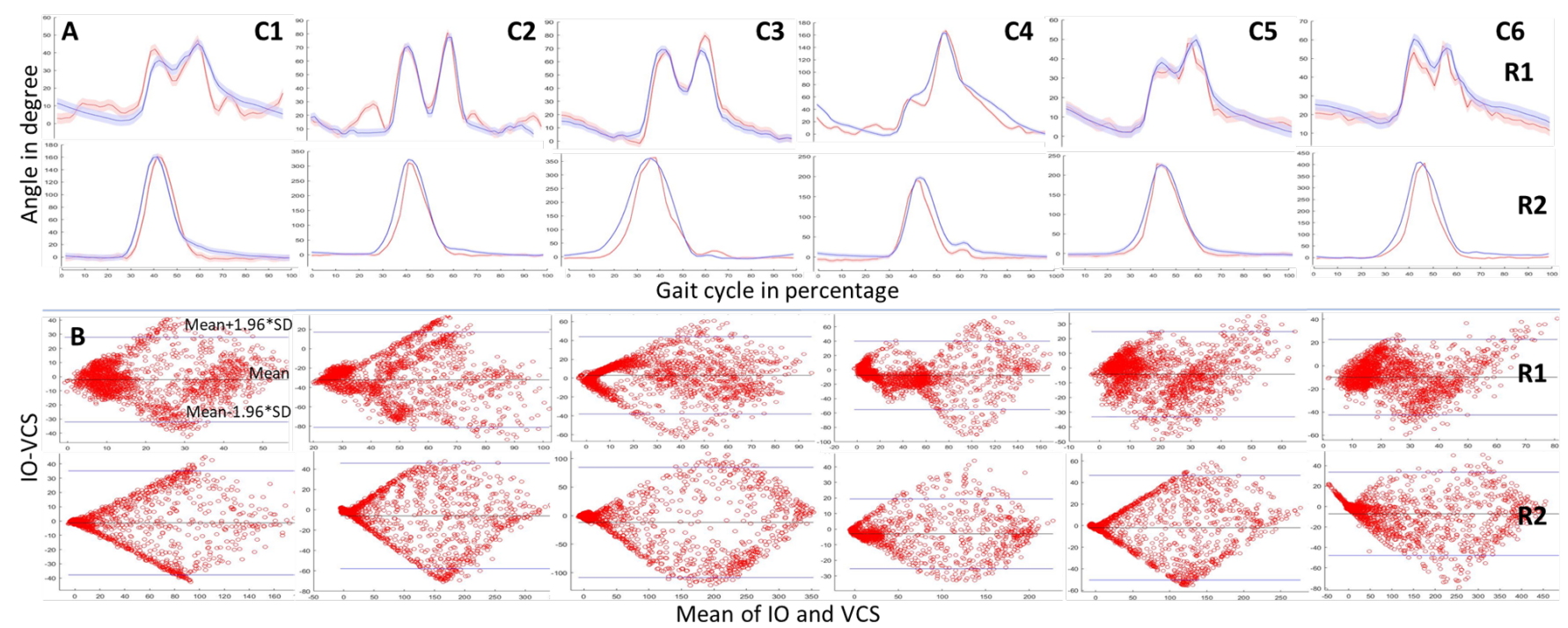

Fig. 6. A: Sample plots of toe clearance (TC) and heel clearance (HC) at various walking speeds and foot landing strategies for one subject. The blue curves correspond to values measured from the video capturing system (VCS), and the red curves correspond to values measured from the instrumented outsole (IO). B: Sample Bland-Altman plots for toe clearance (TC) and heel clearance (HC) at various walking speeds and foot landing strategies for one subject. The Bland-Altman plot is computed for every gait cycle instance throughout the trials. X-value of a point in the plot represents the mean of values measured from IO and VCS. Moreover, the Y-value of a point in the plot represents the difference of values measured from IO and VCS, i.e., IO-VCS. The rows R1 and $\mathrm{R} 2$ correspond to TC and $\mathrm{HC}$, respectively. The columns $\mathrm{C} 1, \mathrm{C} 2, \mathrm{C} 3, \mathrm{C} 4, \mathrm{C} 5$, and C6 correspond to slow walking, medium walking, fast walking, posterior $\mathrm{CoP}$ shift walking, normal $\mathrm{CoP}$ shit walking and anterior $\mathrm{CoP}$ shift walking, respectively.

of the instrumentation outside laboratory environments particularly for home-based monitoring. The potential of the present study has been demonstrated via protocol design involving the intervention of the visual feedback paradigm to simulate the 
different walking patterns of the $\mathrm{CP}$ patients. However, we believe that the actual replication of the walking pattern of the $\mathrm{CP}$ patient was not feasible and therefore in future the recruitment of the $\mathrm{CP}$ patient and testing our instrumentation will be more practical. The toe clearance which is the important parameter for the consideration of the chances of the fall would be integrated in the system and the information should be provided with the vibrotactile feedback at the wrist.

\section{CONCLUSION}

A novel instrumented outsole is developed and validated with a video capturing system. The experiments at various walking speeds and simulated $\mathrm{CP}$ gait patterns are performed on eight healthy subjects to validate foot angles in 3-dimensional planes, toe clearance and heel clearance. The results demonstrated the potential of using the proposed outsole for CP patients.

\section{ACKNOWLEDGEMENT}

We would like to acknowledge Mr. Ayush Tripathi for helping us during extensive data collection. We also thank Dr. Lalan Kumar for providing lab apparatus needed for data collection. In addition, we thank students of Indian Institute of Technology for volunteering as the subjects of the experiments.

\section{REFERENCES}

[1] V. T. Inman, H. D. Eberhart et al., "The major determinants in norma and pathological gait," JBJS, vol. 35, no. 3, pp. 543-558, 1953

[2] W. Pirker and R. Katzenschlager, "Gait disorders in adults and the elderly," Wiener Klinische Wochenschrift, vol. 129, no. 3, pp. 81-95, 2017.

[3] R. Squadrone, R. Rodano, J. Hamill, and E. Preatoni, "Acute effect of different minimalist shoes on foot strike pattern and kinematics in rearfoot strikers during running," Journal of sports sciences, vol. 33, no. 11, pp. 1196-1204, 2015.

[4] C. Brown, "Foot clearance in walking and running in individuals with ankle instability," The American journal of sports medicine, vol. 39, no. 8, pp. 1769-1777, 2011.

[5] M. S. Redfern, R. Cham, K. Gielo-Perczak, R. Grönqvist, M. Hirvonen, H. Lanshammar, M. Marpet, C. Y.-C. Pai IV, and C. Powers, "Biomechanics of slips," Ergonomics, vol. 44, no. 13, pp. 1138-1166, 2001.

[6] H.-C. Chen, J. A. Ashton-Miller, N. B. Alexander, and A. B. Schultz, "Stepping over obstacles: gait patterns of healthy young and old adults," Journal of gerontology, vol. 46, no. 6, pp. M196-M203, 1991.

[7] S. L. Colyer, M. Evans, D. P. Cosker, and A. I. Salo, "A review of the evolution of vision-based motion analysis and the integration of advanced computer vision methods towards developing a markerless system," Sports medicine-open, vol. 4, no. 1, pp. 1-15, 2018.

[8] A. Tiwari and D. Joshi, "Template-based insoles for the center of pressure estimation in different foot sizes," IEEE Sensors Letters, vol. 4, no. 8, pp. 1-4, 2020.

[9] A. Peruzzi, U. Della Croce, and A. Cereatti, "Estimation of stride length in level walking using an inertial measurement unit attached to the foot: A validation of the zero velocity assumption during stance," Journal of Biomechanics, vol. 44, no. 10, pp. 1991-1994, 2011.

[10] X. Yun, J. Calusdian, E. R. Bachmann, and R. B. McGhee, "Estimation of human foot motion during normal walking using inertial and magnetic sensor measurements," IEEE transactions on Instrumentation and Measurement, vol. 61, no. 7, pp. 2059-2072, 2012.

[11] A. Arami, N. Saint Raymond, and K. Aminian, "An accurate wearable foot clearance estimation system: Toward a real-time measurement system,” IEEE Sensors Journal, vol. 17, no. 8, pp. 2542-2549, 2017.

[12] C. Moufawad el Achkar, C. Lenoble-Hoskovec, K. Major, A. ParaschivIonescu, C. Büla, and K. Aminian, "Instrumented shoes for real-time activity monitoring applications," in Nursing Informatics 2016. IOS Press, 2016, pp. 663-667.
[13] F. B. van Meulen, D. Weenk, J. H. Buurke, B.-J. F. van Beijnum, and P. H. Veltink, "Ambulatory assessment of walking balance after stroke using instrumented shoes," Journal of neuroengineering and rehabilitation, vol. 13, no. 1, pp. 1-10, 2016.

[14] C. Moufawad El Achkar, C. Lenoble-Hoskovec, A. Paraschiv-Ionescu, K. Major, C. Büla, and K. Aminian, "Physical behavior in older persons during daily life: Insights from instrumented shoes," Sensors, vol. 16, no. 8 , p. 1225,2016

[15] N. Kitagawa and N. Ogihara, "Estimation of foot trajectory during human walking by a wearable inertial measurement unit mounted to the foot," Gait \& posture, vol. 45, pp. 110-114, 2016.

[16] C. M. El Achkar, C. Lenoble-Hoskovec, A. Paraschiv-Ionescu, K. Major, C. Büla, and K. Aminian, "Instrumented shoes for activity classification in the elderly," Gait \& posture, vol. 44, pp. 12-17, 2016.

[17] N. Carbonaro, F. Lorussi, and A. Tognetti, "Assessment of a smart sensing shoe for gait phase detection in level walking," Electronics, vol. 5 , no. 4, p. 78, 2016

[18] S.-S. Lee, S. T. Choi, and S.-I. Choi, "Classification of gait type based on deep learning using various sensors with smart insole," Sensors, vol. 19, no. 8 , p. $1757,2019$.

[19] R. Das, N. Hooda, and N. Kumar, "A novel approach for real-time gait events detection using developed wireless foot sensor module," IEEE Sensors Letters, vol. 3, no. 6, pp. 1-4, 2019.

[20] U. Sunarya, Y. Sun Hariyani, T. Cho, J. Roh, J. Hyeong, I. Sohn, S. Kim, and C. Park, "Feature analysis of smart shoe sensors for classification of gait patterns," Sensors, vol. 20, no. 21, p. 6253, 2020.

[21] A. Prado, X. Cao, X. Ding, and S. K. Agrawal, "Prediction of gait cycle percentage using instrumented shoes with artificial neural networks," in 2020 IEEE International Conference on Robotics and Automation (ICRA). IEEE, 2020, pp. 2834-2840.

[22] B. Fan, Q. Li, and T. Liu, "Accurate foot clearance estimation during level and uneven ground walking using inertial sensors," Measurement Science and Technology, vol. 31, no. 5, p. 055106, 2020.

[23] J. Hannink, M. Ollenschläger, F. Kluge, N. Roth, J. Klucken, and B. M. Eskofier, "Benchmarking foot trajectory estimation methods for mobile gait analysis," Sensors, vol. 17, no. 9, p. 1940, 2017.

[24] M. Benoussaad, B. Sijobert, K. Mombaur, and C. Azevedo Coste, "Robust foot clearance estimation based on the integration of footmounted imu acceleration data," Sensors, vol. 16, no. 1, p. 12, 2016.

[25] P. Chinmilli, S. Redkar, W. Zhang, and T. Sugar, "A review on wearable inertial tracking based human gait analysis and control strategies of lower-limb exoskeletons," Int. Robot. Autom. J, vol. 3, no. 7, p. 00080, 2017.

[26] N. F. Ribeiro and C. P. Santos, "Inertial measurement units: A brief state of the art on gait analysis," in 2017 IEEE 5th Portuguese Meeting on Bioengineering (ENBENG). IEEE, 2017, pp. 1-4.

[27] A. Tiwari and D. Joshi, "Design and validation of a real-time visual feedback system to improve minimum toe clearance (mtc) in transfemoral amputees," IEEE Transactions on Neural Systems and Rehabilitation Engineering, vol. 29, pp. 1711-1722, 2021.

[28] Y. Wahab and N. A. Bakar, "Gait analysis measurement for sport application based on ultrasonic system," in 2011 IEEE 15th international symposium on consum. er electronics (ISCE). IEEE, 2011, pp. 20-24.

[29] T. Ishikawa and T. Murakami, "Real-time foot clearance and environment estimation based on foot-mounted wearable sensors," in IECON 2018-44th Annual Conference of the IEEE Industrial Electronics Society. IEEE, 2018, pp. 5475-5480.

[30] M. M. van der Krogt, D. J. Bregman, M. Wisse, C. A. Doorenbosch, J. Harlaar, and S. H. Collins, "How crouch gait can dynamically induce stiff-knee gait," Annals of biomedical engineering, vol. 38, no. 4, pp. 1593-1606, 2010.

[31] R. N. Hensinger, Standards in pediatric orthopedics: tables, charts, and graphs illustrating growth. Lippincott Williams \& Wilkins, 1986.

[32] B. Danino, S. Erel, M. Kfir, S. Khamis, R. Batt, Y. Hemo, S. Wientroub, and S. Hayek, "Influence of orthosis on the foot progression angle in children with spastic cerebral palsy," Gait \& posture, vol. 42, no. 4, pp. 518-522, 2015

[33] S. Armand, G. Decoulon, and A. Bonnefoy-Mazure, "Gait analysis in children with cerebral palsy," EFORT open reviews, vol. 1, no. 12, pp. 448-460, 2016.

[34] T. Hoenig, T. Rolvien, and K. Hollander, "Footstrike patterns in runners: Concepts, classifications, techniques, and implications for running-related injuries." German Journal of Sports Medicine/Deutsche Zeitschrift fur Sportmedizin, vol. 71, no. 3, 2020.

[35] A. R. Altman and I. S. Davis, "A kinematic method for footstrike pattern detection in barefoot and shod runners," Gait \& posture, vol. 35, no. 2, pp. 298-300, 2012. 
[36] P. R. Cavanagh and M. A. Lafortune, "Ground reaction forces in distance running," Journal of biomechanics, vol. 13, no. 5, pp. 397-406, 1980.

[37] A. R. De Asha and J. G. Buckley, "The effects of walking speed on minimum toe clearance and on the temporal relationship between minimum clearance and peak swing-foot velocity in unilateral transtibial amputees," Prosthetics and orthotics international, vol. 39, no. 2, pp. 120-125, 2015.

[38] L. S. Law and C. Y. Webb, "Gait adaptation of children with cerebral palsy compared with control children when stepping over an obstacle," Developmental medicine and child neurology, vol. 47, no. 5, pp. 321328, 2005.

[39] C. Bunce, "Correlation, agreement, and bland-altman analysis: statistical analysis of method comparison studies," American journal of ophthalmology, vol. 148, no. 1, pp. 4-6, 2009.

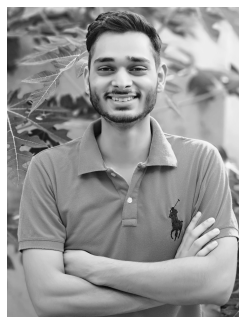

Rishabh Bajpai is currently a $\mathrm{PhD}$ scholar at the Centre for Biomedical Engineering, Indian Institute of Technology (IIT) Delhi, and All India Institute of Medical Sciences (AIIMS) Delhi, India. He received his B.Tech degree in Mechanical Engineering from PDPM Indian Institute of Information Technology, Design and Manufacturing Jabalpur, Madhya Pradesh, India. He has been working in the area of Brain Computer Interface, Rehabilitation engineering, Neuroscience, Electroencephalography and development of biomedical instrumentation for applications specific to assistive devices for disabled.

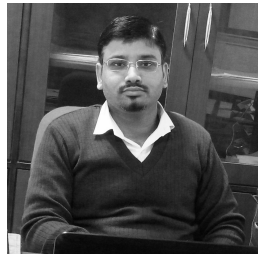

Deepak Joshi is a faculty at Biomedical Engineering at IIT Delhi and All India Institute of Medical Sciences Delhi. He received his $\mathrm{PhD}$ in biomedical engineering from Indian Institute of Technology (IIT) Delhi. He has been working in the area of neuroprosthetics and neurorehabilitation for last nearly fourteen years. Before joining to IIT Delhi, he worked at various places including National University of Singapore, Newcastle University UK, and University of Oregon, USA. Dr. Joshi's current research work combines experimental and computational techniques to understand the neural correlates during balancing and seamless transitions during walking. Besides that, he is actively engaged in projects related to development of wearable devices for applications specific to diagnosis of neuromuscular disorders, assistive devices for elderly and disabled, and biofeedback for rehabilitation in stroke patients.

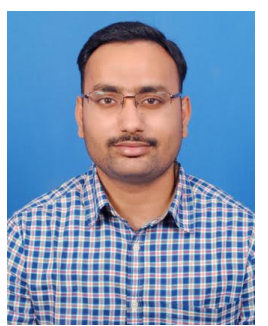

Ashutosh Tiwari received B. Tech. and M. Tech. in Biomedical Engineering from Vellore Institute of Technology, Vellore, India in 2013 and Indian Institute of Technology Bombay, India, in 2015 respectively. He is currently perusing his Ph.D. degree in Centre for Biomedical Engineering, from Indian Institute of Technology Delhi, India. His research interests include Biomechanics, Rehabilitation, Biomedical signal processing, Wearable technologies for gait applications.

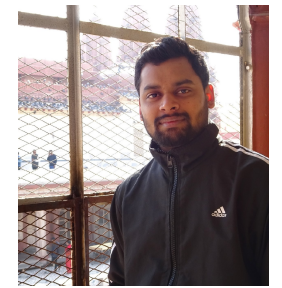

Anant Jain received the M.Tech degree in Biomedical Engineering from National Institute of Technology, Kurukshetra, India, in 2018. He has worked as Research Assistant in the Centre of Biomedical Engineering, IIT Delhi between 2018-19. Since 2019, he has joined as Research Scholar in Department of Electrical Engineering at MSP Lab, IIT Delhi. His research interests include biomedical signal processing, computational neuroscience, Brain computer interface, Human-machine interface, Machine learning $\&$ Deep learning. 